\title{
Jets from Compact X-ray Sources
}

\author{
Nick D. Kylafis ${ }^{1,2}$ \\ ${ }^{1}$ Foundation for Research and Technology - Hellas, Institute of Electronic Structure and Laser, \\ 71110 Heraklion, Crete, Greece \\ ${ }^{2}$ University of Crete, Physics Department, 71003 Heraklion, Crete, Greece \\ email: kylafis@physics.uoc.gr
}

\begin{abstract}
Jets have been observed from both neutron stars and black holes in binary X-ray sources. The neutron star jets are typically 30 times weaker than the black-hole ones. Thus, the second have been studied more extensively. Contrary to common belief, jets from compact $\mathrm{X}$-ray sources are not simply "fireworks" that emit radio waves. I will demonstrate that they play a central role in the observed phenomena in both neutron star and black-hole systems. In particular, for black-hole jets, a simple jet model can explain the very stringent correlations that have been found between the power-law X-ray spectrum and a) the time lag between hard and soft X-rays and $\mathrm{b}$ ) the characteristic frequencies observed in the power spectra. Up to now, no other model has even attempted to explain these correlations. I will present the weaknesses of the model and the improvements that need to be done to it.
\end{abstract}

Keywords. X-ray sources, black holes, neutron stars, jets

\section{Short summary of presentation}

It is generally perceived in our community that jets from compact X-ray sources (neutron stars and black holes) play no other role in the observed phenomena than to exhibit emission of radio waves and ejection of matter that were to fall on the compact object. To explain the X-ray spectrum, most models assume that there is a hot, static, spherical (or nearly spherical) corona near the compact object. Up to now, no physical mechanism has been proposed that creates and sustains such a static corona. Worse than this, it is not questioned whether such a structure can actually exist.

On the other hand, nobody doubts that a jet is always present when the X-ray source is in the so called hard spectral state. Furthermore, nobody doubts that the observd radio spectrum implies the existence of energetic electrons in the jet. The question then naturally arises: Why should one invoke a corona to explain with comptonization the hard X-ray spectrum, when the jet itself can do the job?

In a series of papers ( Reig et al. (2003); Giannios et al. (2004); Giannios (2005); Kylafis et al. (2008)), we demostrated that a simple jet model can explain:

a) The entire spectrum from radio to hard X-rays.

b) The time lags between hard and soft X-rays and their correlation with Fourier frequency.

c) The narrowing of the autocorrelation function with photon energy or equivalently the increase of the rms amplitude of variability with photon energy.

d) The correlation of the hard X-ray power law index with the time lags.

e) The correlation of the hard X-ray power law index with the characteristic Fourier frequencies in the power spectrum.

Furthermore, we predicted and confirmed that the radio emission should correlate positively with the hard X-ray power law index. 
Our model does not reproduce the reflected spectrum from the accretion disk. This is because we assumed, for simplicity, that the velocity of the jet is constant from its base to the top. This is, of course, unnatural. The ejected matter starts with low bulk velocity and it is gradually accelerated to its terminal velocity. We will improve our model and will report our results in the near future.

Acknowledgement: This research has been supported in part by European Union grants 39965 and 206469.

\section{References}

Giannios, D., Kylafis, N. D., \& Psaltis D. 2004, A\& $A, 425,163$

Giannios, D. 2005, A\&\&A, 437, 1007

Kylafis, N. D., Papadakis, I. E., Reig, P., Giannios, D., \& Pooley, G. G. 2008, A $\& A$, 489, 481

Reig, P., Kylafis, N. D., \& Giannios, D. 2003, A $\mathscr{E} A$, 403, L15 